OPEN ACCESS

Edited by:

Guido Favia,

University of Camerino, Italy

Reviewed by:

Jeremy Keith Herren,

International Centre of Insect

Physiology and Ecology (ICIPE),

Kenya

Ewa Anna Chrostek,

Max Planck Institute for Infection

Biology, Germany

${ }^{*}$ Correspondence:

Zhiyong $X$

xizy@msu.edu

Specialty section:

This article was submitted to

Microbial Symbioses,

a section of the journal

Frontiers in Microbiology

Received: 22 April 2020

Accepted: 23 June 2020

Published: 10 July 2020

Citation:

Liang X, Liu J, Bian G and Xi Z

(2020) Wolbachia Inter-Strain

Competition and Inhibition

of Expression of Cytoplasmic

Incompatibility in Mosquito.

Front. Microbiol. 11:1638.

doi: 10.3389/fmicb.2020.01638

\section{Wolbachia Inter-Strain Competition and Inhibition of Expression of Cytoplasmic Incompatibility in Mosquito}

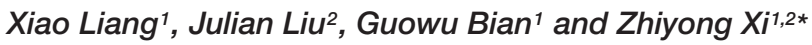 \\ 1 Department of Microbiology and Molecular Genetics, Michigan State University, East Lansing, MI, United States, \\ ${ }^{2}$ Guangzhou Wolbaki Biotech Co., Ltd., Guangzhou, China
}

Successful field trials have been reported as part of the effort to develop the maternally transmitted endosymbiontic bacteria Wolbachia as an intervention agent for controlling mosquito vectors and their transmitted diseases. In order to further improve this novel intervention, artificially transinfected mosquitoes must be optimized to display maximum pathogen blocking, the desired cytoplasmic incompatibility $(\mathrm{Cl})$ pattern, and the lowest possible fitness cost. Achieving such optimization, however, requires a better understanding of the interactions between the host and various Wolbabachia strains and their combinations. Here, we transferred the Wolbachia wMel strain by embryonic microinjection into Aedes albopictus, resulting in the successful establishment of a transinfected line, HM (wAlbAwAlbBwMel), with a triple-strain infection comprising wMel, wAlbA, and wAlbB. Surprisingly, no $\mathrm{Cl}$ was induced when the triply infected males were crossed with the wild-type GUA females or with another triply infected $\mathrm{HC}$ females carrying $w$ Pip, wAlbA, and $w A \mathrm{lbB}$, but specific removal of wAlbA from the $\mathrm{HM}$ (wAlbAwAlbBwMel) line resulted in the expression of $\mathrm{Cl}$ after crosses with lines infected by either one, two, or three strains of Wolbachia. The transinfected line showed perfect maternal transmission of the triple infection, with fluctuating egg hatch rates that improved to normal levels after repeated outcrosses with GUA line. Strain-specific qPCR assays showed that $w \mathrm{Mel}$ and $w A \mathrm{AlbB}$ were present at the highest densities in the ovaries and midguts, respectively, of the HM (wAlbAwAlbBwMel) mosquitoes. These finding suggest that introducing a novel strain of Wolbachia into a Wolbachia-infected host may result in complicated interactions between Wolbachia and the host and between the various Wolbachia strains, with competition likely to occur between strains in the same supergroup.

Keywords: Wolbachia, dengue, cytoplasmic incompatibility, Aedes albopictus, transinfection, inter-strain competition

\section{INTRODUCTION}

A rapid increase in the number of arbovirus diseases transmitted by mosquitoes, such as dengue and Zika, in recent decades has underscored the urgency in developing effective intervention strategies (Velayudhan, 2012; Katzelnick et al., 2017). The insufficiency of traditional control approaches, including vaccines, drugs, and chemical insecticides, has led to significant efforts to develop novel 
vector control methods to combat disease transmission. Rather than using chemical insecticides to directly kill the vector, an approach that is being challenged by the rapid development of insecticide resistance and the negative impacts on both the environment and non-target insect species, these new tools have focused on modifying the mosquito population in a speciesspecific manner, with the goal of either reducing the mosquito's ability to host a pathogen or suppressing (or even eliminating) the mosquito population to break the viral transmission between vector and host (Achee et al., 2015). Among these strategies, Wolbachia-based interventions have recently shown encouraging results in field trials, successfully demonstrating either reduced dengue transmission through Wobachia-induced viral inhibition in the mosquitoes or the elimination of the Aedes vector populations through Wobachia-induced incompatible mating (Hoffmann et al., 2011; Mains et al., 2016; Nazni et al., 2019; Zheng et al., 2019; Crawford et al., 2020).

Estimated to infect $>65 \%$ of all insect species, Wolbachia are maternally transmitted endosymbiotic bacteria belong to the order Rickettsiales and family Anaplasmataceae (Werren et al., 2008). Designated based on their naturally associated host species and divided into eight supergroups, different Wolbachia strains can interact with their hosts in their own manner, with phenotypes determined by the genetic background of both Wolbachia and the host, as well as the environment (McGraw et al., 2002; Werren et al., 2008; Ross et al., 2017). In the mosquito and many other insects, Wolbachia causes a reproduction alteration known as cytoplasmic incompatibility (CI), in which early embryonic death occurs when the Wolbachiainfected male mates with an uninfected female or a female carrying a different strain of Wolbachia. The CI can be rescued, resulting in compatible mating, if the Wolbachia strain carried by the male is also present in the female. Recent studies have shown that two $C I$ determination genes, cifA and cifB, in Wolbachia modify the sperm development to induce CI, but only cifA mediates CI rescue in females (or eggs; LePage et al., 2017; Shropshire et al., 2018; Beckmann et al., 2019; Chen et al., 2019). However, it is still unknown how these CI factors interact with their host targets and how the CI determination factors of different Wolbachia strains interact with each other to induce CI expression in a host with a Wolbachia superinfection.

Since the ability to generate novel Wolbachia symbiosis (transinfection) in mosquitoes was first developed through embryonic microinjection (Xi et al., 2005a,b, 2006), a number of transinfected mosquito lines carrying different Wolbachia strains have been established and characterized, with the goal of using them for disease control (Xi et al., 2005b, 2006; McMeniman et al., 2009; Walker et al., 2011; Blagrove et al., 2012; Joubert et al., 2016; Ant et al., 2018; Ross et al., 2019). Many of these transinfected mosquito lines show different levels of resistance to dengue, Zika, and Chikungunya viruses, with the strength of the viral inhibition being associated with the density of Wolbachia in somatic tissues such as the midgut and salivary glands, where the viruses reside, migrate, and replicate. Whereas transinfected lines with each of three Wolbachia strains - wMel, wAlbB, and $w$ Pip - have been well characterized and successfully tested in field trials (Hoffmann et al., 2011; Nazni et al., 2019; Zheng et al., 2019), significant interest remains in developing improved transinfected lines with maximal viral blocking and optimal fitness under field conditions in order to reach the highest efficiency in disease control or to be able to replace the released lines if viruses develop resistance to the released strains in the future (Ross et al., 2019).

Naturally carrying two Wolbachia strains, wAlbA and $w \mathrm{AlbB}$, Aedes albopictus is the world's most invasive mosquito vector and an epidemiologically important vector for many arboviruses. As the density of these two native Wolbachia is too low to induce viral inhibiton in Ae. albopictus (Lu et al., 2012), efforts have been made to introduce novel strains into this mosquito species to develop transinfected lines that are both incompatible with the wild-type line and resistant to viruses (Blagrove et al., 2013; Zheng et al., 2019). Experiments are often designed by either directly adding a novel strain to Ae. albopictus to generate a superinfection (Fu et al., 2010; Suh et al., 2016; Ant and Sinkins, 2018; Zheng et al., 2019) or replacing the native Wolbachia with a novel strain, by removing the native Wolbachia with an antibiotic and then introducing the novel strain (Xi et al., 2006; Blagrove et al., 2012). The first approach results in a triple infection to induce an unidirectional CI with wild-type mosquitoes (Fu et al., 2010; Zheng et al., 2019), with the advantage that Wolbachia invade and spread into the population more effectively than does the second (replacement) approach, which often induces a bi-directional CI (Xi et al., 2006; Blagrove et al., 2012). However, for a host with a triple-strain infection, the outcome of the transinfection is difficult to predict, given the complicated interactions between the various Wolbachia strains and between Wolbachia and the host (Suh et al., 2016; Ant and Sinkins, 2018). When a tripe-strain infection comprising $w \mathrm{Mel}, w \mathrm{AlbA}$, and $w \mathrm{AlbB}$ was previously established in Ae. albopictus, very low egg hatch rates were observed in both the self-cross of the transinfected line and the compatible cross of the transinfected females with wild-type males (Ant and Sinkins, 2018), suggesting that the ability of this Wolbachia triple-strain infection to recue CI modification was compromised due to unknown interstrain interactions.

We previously developed the transinfected Ae. albopictus line $\mathrm{HC}$, featuring another triple infection with $w$ Pip, $w$ AlbA, and $w$ AlbB (Zheng et al., 2019). The HC line induces complete unidirectional CI in crosses with the wild-type line, with intact ability of $\mathrm{HC}$ females to rescue CI when mated with either wildtype or HC males (Zheng et al., 2019). In the present study, we have introduced $w \mathrm{Mel}$ into Ae. albopictus and generated the transinfected line, HM ( $w A l b A w A l b B w \mathrm{Mel}$ ), infected with $w \mathrm{Mel}, w \mathrm{AlbA}$, and $w \mathrm{AlbB}$. The transinfected line show complete efficiency in maternal transmission of the triple infection, with $w \mathrm{Mel}$ showing the highest density in ovaries. Multiple crosses showed that the ability of $w \mathrm{Mel}$ to induce CI was blocked by $w \mathrm{AlbA}$ in the HM ( $w \mathrm{AlbA} w \mathrm{AlbB} w \mathrm{Mel})$ line and that double infection with $w \mathrm{Mel}$ and $w \mathrm{AlbB}$ induced a high level of $\mathrm{CI}$ in crosses with the lines infected with either a single, double, or triple infection. 


\section{MATERIALS AND METHODS}

\section{Mosquito Lines and Maintenance}

Two wild-type Ae. albopictus lines, HOU (Xi et al., 2005a) and GUA (Zheng et al., 2019), carrying a native superinfection with $w \mathrm{AlbA}$ and $w \mathrm{AlbB}$ were used in this study. Two transinfected Ae. albopictus lines, $\mathrm{HB}$ and $\mathrm{HC}$, carrying a single $w \mathrm{AlbB}$ infection and triple infection with $w \mathrm{Mel}, w \mathrm{AlbA}$, and $w \mathrm{AlbB}$, respectively, had been generated previously (Xi et al., 2005a; Zheng et al., 2019) and were used in the CI crosses. The transinfected Aedes aegypti MGYP2 line (Walker et al., 2011), carrying $w \mathrm{Mel}$, was used as a donor to generate the HM ( $w \mathrm{AlbA} w \mathrm{AlbB} w \mathrm{Mel}$ ) line.

All the mosquito lines were maintained on a $10 \%$ sugar solution at $27 \pm 1{ }^{\circ} \mathrm{C}$ and $80 \pm 10 \%$ relative humidity, with a 12:12 h light:dark photoperiod, according to standard rearing procedures. For routine colony maintenance and experimental studies, female mosquitoes were provided with either human (for the MGYP2 line) or sheep (for the other lines) blood at day-7 post-eclosion, and eggs were collected 2 days post-blood meal.

\section{Transinfection to Generate the HM (wAlbAwAlbBwMel) Line}

The HM ( $w \mathrm{AlbA} w \mathrm{AlbB} w \mathrm{Mel})$ line was generated by transfer of $w \mathrm{Mel}$ from Ae. aegypti MGYP2 to Ae. albopictus HOU using embryonic microinjection according to the approach described previously (Xi et al., 2005a,b). In brief, cytoplasm from donor embryos was transferred into the posterior of 60-90-min-old recipient embryos using an IM300 microinjector (Narishige Scientific). After injection, the embryos were incubated at $85 \%$ relative humidity and $27^{\circ} \mathrm{C}$ for $1 \mathrm{~h}$, then transferred to wet filter paper. Embryos were allowed to mature for 5-7 days before hatching. Females (G0) developing from the surviving embryos were isolated and mated with HOU males. After blood-feeding and oviposition, G0 females were tested for $w \mathrm{Mel}$ infection by PCR using strain-specific primers as described below. G1 females were again crossed with HOU males, blood-fed, isolated, and allowed to oviposit. The offspring from $w \mathrm{Mel}$-positive G1 were selected for the next screen, and this process was repeated until the $w \mathrm{Mel}$ maternal transmission rate reached $100 \%$. Diagnosis of Wolbachia wAlbA and $w \mathrm{AlbB}$ was also performed to ensure that the transinfected line carried the triple infection.

\section{PCR Assays of Wolbachia Infection}

Primers were designed for strain-specific diagnosis of four different strains on the basis of the sequence of the gene encoding the Wolbachia surface protein wsp. The primers for $w$ AlbA were: forward $5^{\prime}$-GTGTTGGTGCAGCGTATGTC$3^{\prime}$; reverse 5'-GCACCAGTAGTTTCGCTATC-3'. The primers for $w$ AlbB were: forward $5^{\prime}$-ACGTTGGTGGTGCAACATTTG$3^{\prime}$; reverse $5^{\prime}$-TAACGAGCACCAGCATAAAGC-3'. The primers for $w \mathrm{Mel}$ were: forward $5^{\prime}$-CCTTTGGAACCCGCTGTGAATG$3^{\prime}$; reverse $5^{\prime}$-GCCTGCATCAGCAGCCTGTC-3'. The primers for $w$ Pip were: forward $5^{\prime}$-TATTTCCCACTATATCCCTTC- $3^{\prime}$; reverse $5^{\prime}$-GGATTTGACCTTTCCGGC-3'. The primers given below for mosquito rps6 have been reported previously (MolinaCruz et al., 2005): forward 5'-CGTCGTCAGGAACGTATTCG$3^{\prime}$; and reverse $5^{\prime}$-TCTTGGCAGCCTTGACAGC-3'. Standard curves were generated for each of the genes listed above to convert the $\mathrm{Ct}$ value from quantitative PCR (qPCR) to the copy number of target sequences.

Genomic DNA was extracted from the samples using a Thermo Scientific Phire Animal Tissue Direct PCR Kit (F$140 \mathrm{WH})$. Samples were pre-treated in $20 \mu \mathrm{l}$ of dilution buffer with $0.5 \mu \mathrm{l}$ DNARelease Additive. The reaction mixture contained $10 \mu \mathrm{l}$ 2X Phire Animal Tissue PCR Buffer, $0.4 \mu \mathrm{l}$ Phire Hot Start II DNA Polymerase, $0.2 \mu \mathrm{l}$ of both the

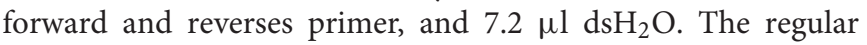
PCR conditions were: initial denaturation at $98^{\circ} \mathrm{C}$ for $6 \mathrm{~min}$, followed by 40 cycles of $5 \mathrm{~s}$ at $98^{\circ} \mathrm{C}, 5 \mathrm{~s}$ at $56^{\circ} \mathrm{C}$, and $45 \mathrm{~s}$ at $72^{\circ} \mathrm{C}$. qPCR was performed using a QuantiTect SYBR Green PCR Kit (Qiagen) and ABI Detection System ABI Prism 7000 (Applied Biosystems, Foster City, CA, United States). Samples were homogenized in $100 \mu \mathrm{l} 1 \times$ STE buffer and incubated with $4 \mu \mathrm{l}$ of roteinase $\mathrm{K}$ at $55^{\circ} \mathrm{C}$ for $1 \mathrm{~h}$, followed by $97^{\circ} \mathrm{C}$ for $5 \mathrm{~min}$.

\section{Tetracycline Treatment of the HM (wAlbAwAlbBwMel) Line to Generate the HM2 (wAlbBwMel) Line With a Double Infection of $w \mathrm{Mel}$ and $w \mathrm{AlbB}$}

Once the HM ( $w$ AlbAwAlbBwMel) mosquitoes had emerged as adults (day 0 ), they were provided with $0.5 \mathrm{mg} / \mathrm{ml}$ tetracycline $\mathrm{HCl}$ in a $10 \%$ sugar solution. This solution was replaced with a $10 \%$ sugar solution from day 3 or 4 , and a blood meal was provided on day 7. Two days after the blood feeding, the mosquitoes were provided with oviposition cups containing wet filter paper. These treatments were repeated for four generations. At G3, after blood-feeding, the females were isolated for oviposition. After their eggs collected, individual isofemales were sacrificed to extract genomic DNA, and a PCR assay was used to identify each of the three Wolbachia strains. Only the eggs from females showing a double infection with $w \mathrm{Mel}$ and $w \mathrm{AlbB}$ were allowed to hatch to establish the line. The isofemale selection described above was repeated at G5 to ensure the removal of $w \mathrm{AlbA}$, and the resulting HM2 $(w \mathrm{AlbB} w \mathrm{Mel})$ line carried only the double infection with $w \mathrm{Mel}$ and $w$ AlbB.

\section{Experimental Crosses to Determine CI}

Cytoplasmic incompatibility assays were conducted as previously described (Xi et al., 2005a,b). A total of 10 virgin males were mated with 10 virgin females in five replicate cages for each cross. A blood meal was provided to the females at day 7 posteclosion. Two days after the blood meal, eggs were collected using oviposition cups containing wet filter paper, which was subsequently desiccated for 7 days at $27^{\circ} \mathrm{C}$ and $80 \%$ relative humidity. The eggs were counted and then hatched in water containing $6 \%(\mathrm{~m} / \mathrm{v})$ bovine liver powder. Larvae were counted at the L2-L3 stage to record the hatch rate. 


\section{Statistical Analysis}

All data were statistically analyzed by GraphPad Prism 5.0 software. ANOVA and Tukey's multiple comparisons test were used to compare egg hatching in CI cross experiment and density of each Wolbachia strain in mosquito salivary glands, midguts, and ovaries.

\section{RESULTS}

\section{Generation of the Ae. albopictus Transinfected Line With a Triple Wolbachia Infection: wMel, wAlbA, and wAlbB}

The ability of a single $w \mathrm{Mel}$ infection to inhibit arbovirus transmission in both Ae. aegypti and Ae. albopictus (Walker et al., 2011; Blagrove et al., 2012) motivated us to test whether a triple infection with $w \mathrm{Mel}, w \mathrm{AlbA}$, and $w \mathrm{AlbB}$ could be established in Ae. albopictus to produce enhanced viral blocking effects for disease control, and whether there was competition among the various Wolbachia strains that might affect the nature of the symbiosis between Wolbachia and its mosquito host. The cytoplasm of wMel-infected Ae. aegypti (MGYP2) embryos (Walker et al., 2011) was transferred by microinjection into embryos of the Ae. albopictus HOU line with a native superinfection of $w \mathrm{AlbA}$ and $w \mathrm{AlbB}$ (Figure 1A). The virgin females (G0) developed from embryos surviving the microinjection were outcrossed with HOU males to produce offspring (G1). A total of $18 \mathrm{Gl}$ isofemales were outcrossed with HOU males. After their eggs (G2) were collected, PCR assay was used to diagnose the Wolbachia strain profile in these females, with 15 of 18 isofemales (83\%) being seen to carry the triple Wolbachia infection (Figures 1B,C); the offspring of the females without a triple infection were discarded. Among the G2 offspring of these triply infected mothers, 18 of 20 (90\%) males and 15 of 20 (75\%) females maintained a triple Wolbachia infection. Without further screening, the offspring from the triply infected G2 females were then pooled together to establish a new transinfected line, hereafter referred to as HM ( $w \mathrm{AlbA} w \mathrm{AlbB} w \mathrm{Mel}$ ). At G3 and G4, we randomly selected 20 and 10 individuals, respectively, for PCR assay. All of the tested mosquitoes carried a triple infection, indicating a 100\% maternal transmission efficiency. Subsequently, the infection status of the HM ( $w$ AlbA $w$ AlbB $w \mathrm{Mel})$ line was monitored every other generation from G8 to G24, and all the tested samples $(n=126)$ were positve, confirming the stability of the triple infection in the HM ( $w \mathrm{AlbA} w \mathrm{AlbB} w \mathrm{Mel}$ ) line (Figure 1C). These results suggest that $w \mathrm{Mel}$ can coexist with $w \mathrm{AlbA}$ and $w \mathrm{AlbB}$ to exhibit symbiosis within Ae. albopictus.

\section{Failure of the HM (wAlbAwAlbBwMel) Line to Induce $\mathrm{CI}$ When Crossed With Wild-Type or Transinfected Lines}

The ability to induce $\mathrm{CI}$ is a key feature that is required in order to develop Wolbachia-based strategies for mosquitoborne disease control. We therefore set up a series of reciprocal crosses among the HM ( $w \mathrm{AlbA} w \mathrm{AlbB} w \mathrm{Mel}$ ), GUA, and $\mathrm{HC}$ lines to measure the relative strength of the $w$ Mel-mediated CI expression. All compatible crosses yielded egg hatch rates ranging from 51 to $56 \%$ (Table 1). Unexpectedly, two presumably incompatible crosses, matings between $\mathrm{HM}$ ( $w \mathrm{AlbA} w \mathrm{AlbB} w \mathrm{Mel}$ ) males and either GUA or HC females, resulted in high egg hatch rates (46.7 and $43.2 \%$, respectively), indicating compatible mating between them. In contrast, consistent with the ability of $\mathrm{HC}$ males to induce a strong $\mathrm{CI}$ when crossed with GUA females (Zheng et al., 2019), near-complete CI was observed in the crosses between $\mathrm{HC}$ males and $\mathrm{HM}$ $(w \mathrm{AlbA} w \mathrm{AlbB} w \mathrm{Mel})$ females (Table 1). These results indicate that the ability of $w \mathrm{Mel}$ to induce $\mathrm{CI}$, as observed previously (Walker et al., 2011; Blagrove et al., 2012), is blocked in the $\mathrm{HM}(w \mathrm{AlbA} w \mathrm{AlbB} w \mathrm{Mel})$ line when it co-exists with $w \mathrm{AlbA}$ and $w$ AlbB.

\section{Cl Induction by $w$ Mel After Removal of wAlbA From the HM (wAlbAwAlbBwMel) Line in Ae. albopictus}

In order to understand whether the ability of $w \mathrm{Mel}$ to induce $\mathrm{CI}$ in the HM ( $w \mathrm{AlbA} w \mathrm{AlbB} w \mathrm{Mel})$ line is being blocked by the other two native Wolbachia strains, we treated the HM $(w \mathrm{AlbA} w \mathrm{AlbB} w \mathrm{Mel})$ line with a subdose of tetracycline for four generations and monitored the infection profile by strain-specific PCR from G3 to G5 after tetracycline treatment (Figures 2AC). This treatment resulted in the specific removal of $w \mathrm{AlbA}$ from the HM ( $w \mathrm{AlbA} w \mathrm{AlbB} w \mathrm{Mel})$ line and establishment of the HM2 ( $w$ AlbBwMel) line, with a double infection of $w \mathrm{Mel}$ and $w$ AlbB (Figure 2C). CI crosses were then performed using HM2 (wAlbBwMel), GUA, HC, and an Ae. albopictus $\mathrm{HB}$ line with a single $w$ AlbB infection. Strikingly, we observed a strong, although not complete, CI when HM2 ( $w \mathrm{AlbB} w \mathrm{Mel}$ ) males were crossed with GUA, HC, or HB females (Table 2). As expected, HM2 ( $w \mathrm{AlbB} w \mathrm{Mel})$ induced bi-directional CI when crossed with the GUA and HC lines, but uni-directional $\mathrm{CI}$ when crossed with the $\mathrm{HB}$ line. Among all of these incompatible crosses, $\mathrm{HC}$ males induced the highest level of CI, with $100 \%$ embryonic death. These results indicate that $w$ AlbA may block the expression of $\mathrm{CI}$ by $w \mathrm{Mel}$ in the $\mathrm{HM}$ $(w \mathrm{AlbA} w \mathrm{AlbB} w \mathrm{Mel})$ line.

\section{Introduction of a New Host Genetic Background Into the HM (wAlbAwAlbBwMel) Line to Increase Its} Fitness

The newly established HM ( $w$ AlbAwAlbBwMel) line suffered from a strong fitness cost associated with the triple-strain infection, with an extremely low egg hatch rate ranging from 1 to $12 \%$ between G2 and G5 (Figure 3). Therefore, we outcrossed $\mathrm{HM}(w \mathrm{AlbA} w \mathrm{AlbB} w \mathrm{Mel})$ females with HOU males to remove the potential inbreeding effect, which has been observed to cause a low egg hatch rate in previous transinfected lines (Xi et al., 2005a, 2006). The egg hatch rate increased to $60 \%$ at G6, then dropped to 12 and 6\% at G10 and G12, respectively (Figure 3). From G13 to G27, the egg hatch rate continued 


\section{A Add wMel into HOU by embryo microinjection:

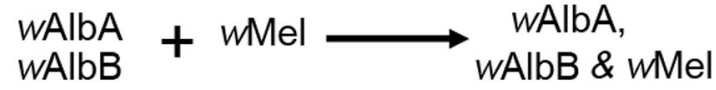

B

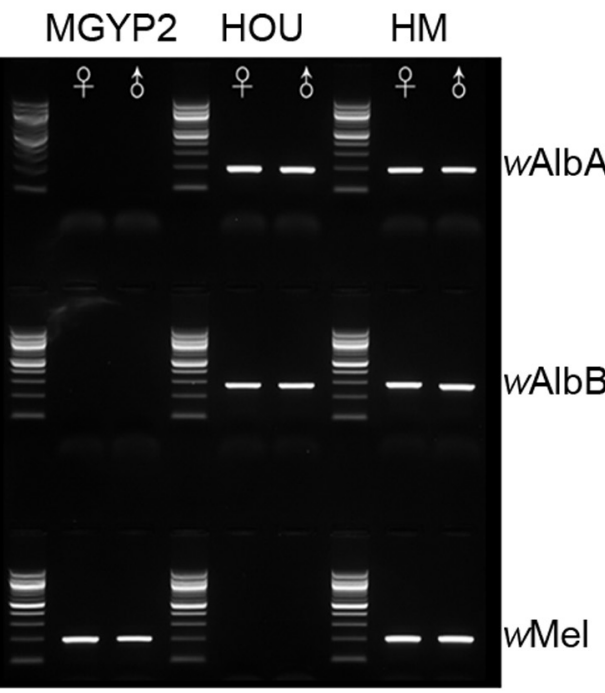

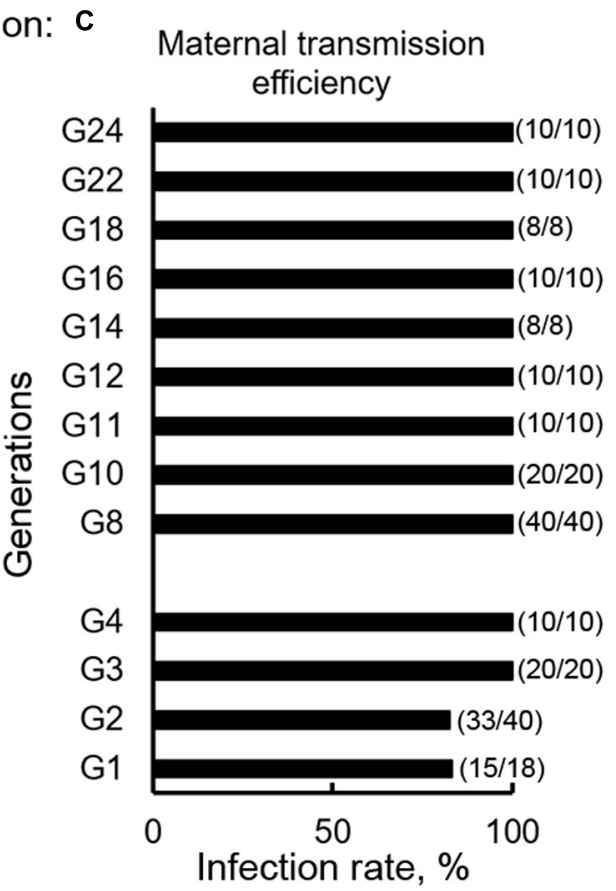

FIGURE 1 | Establishment of the transinfected Ae. albopictus HM (wAlbAwAlbBwMel) line with a triple infection. (A) Schematic diagram of the experimental design to establish the HM (wAlbAwAlbBwMel) line with a Wolbachia triple-strain infection. (B) Representative results of the strain-specific amplification of the three Wolbachia strains, wMel, wAlbA, and wAlbB, in a PCR assay. MGYP2, the transinfected Aedes aegypti line carrying wMel, serving as a donor during the embryonic microinjection experiment in this study. $\mathrm{HOU}$, Ae. albopictus $\mathrm{HOU}$ line carrying wAlbA and wAlbB, serving as the recipient. HM, transinfected Ae. albopictus line carrying the triple infection wMel, wAlbA, and wAlbB. (C) Maternal transmission efficiency was monitored by randomly selecting individuals from each generation, as indicated, and diagnosis of Wolbachia infection by PCR using strain-specific primers. The infection rate was calculated as the percentage of positive individuals in the tested samples at the designated generation.

TABLE 1 | Results of $\mathrm{Cl}$ crosses among the HM (wAlbAwAlbBwMel), GUA, and HC lines.

\begin{tabular}{|c|c|c|c|c|}
\hline \multirow[t]{2}{*}{ Expected $\mathrm{Cl}$ type } & \multirow[t]{2}{*}{ Cross $\left(\phi \times \sigma^{7}\right)$} & \multicolumn{2}{|c|}{ Infection type } & \multirow[t]{2}{*}{ Percent egg hatch } \\
\hline & & Female & Male & \\
\hline \multirow[t]{3}{*}{ Incompatible } & $\mathrm{HC} \times \mathrm{HM}$ & wAlbA,wAlbB, wPip & wAlbA,wAlbB, wMel & $43.2 \pm 11.6 \mathrm{a}$ \\
\hline & $\mathrm{HM} \times \mathrm{HC}$ & $w A l b A, w A l b B, w \mathrm{Mel}$ & wAlbA,wAlbB, wPip & $0.01 \pm 0.04 b$ \\
\hline & $\mathrm{GUA} \times \mathrm{HM}$ & $w A l b A, w A l b B$ & $w A l b A, w A l b B, w$ Mel & $46.7 \pm 7.8 \mathrm{c}$ \\
\hline \multirow[t]{3}{*}{ Compatible } & $\mathrm{HM} \times \mathrm{GUA}$ & wAlbA,wAlbB, wMel & wAlbA,wAlbB & $55.5 \pm 17.8 \mathrm{c}$ \\
\hline & $\mathrm{HM} \times \mathrm{HM}$ & wAlbA,wAlbB, wMel & $w A l b A, w A l b B, w \mathrm{Mel}$ & $55.1 \pm 9.0 \mathrm{c}$ \\
\hline & $\mathrm{HC} \times \mathrm{HC}$ & $w A l b A, w A l b B, w$ Pip & $w A l b A, w A l b B, w$ Pip & $51.3 \pm 11.7 \mathrm{c}$ \\
\hline
\end{tabular}

${ }^{\star}$ Expressed as the mean for 15 replicates/cross type \pm standard deviation. Different letters following the data indicate significant differences ( $P$ < 0.001$)$ by ANOVA-Tukey's multiple comparison test.

fluctuating and varied from 8 to $65 \%$, indicating that the low egg hatch rate may not be only caused by inbreeding; the maladaptation of the novel triple-strain infection to the HOU genetic background may also have contributed to this fitness cost. Thus, at G16, we started to outcross the HM ( $w \mathrm{AlbA} w \mathrm{AlbB} w \mathrm{Mel}$ ) females with males of the GUA strain, a wild-type Ae. albopictus recently collected from the field in Guangzhou, China (Zheng et al., 2019). A steady increase in the egg hatch rate of the outcrossed HM ( $w \mathrm{AlbA} w \mathrm{AlbB} w \mathrm{Mel}$ ) line was then observed, from $21 \%$ at G19 to $84 \%$ at G27 (Figure 3), the higher level being similar to that in the GUA strain. Thus, it appears that the GUA genetic background is able to overcome the triple infection-associated decrease in egg hatch rate in the $\mathrm{HM}$ ( $w \mathrm{AlbA} w \mathrm{AlbB} w \mathrm{Mel})$ line.

\section{$w$ Mel Distribution in Both the Somatic and Germline Tissues in the HM (wAlbAwAlbBwMel) Line}

Wolbachia tissue tropism is an important determining factor underlying its viral blocking effect and maternal transmission. We first compared the densities of the three Wolbachia strains, $w \mathrm{Mel}, w \mathrm{AlbA}$, and $w \mathrm{AlbB}$, in somatic tissues (salivary glands and midgut) and germline tissues (ovaries) of HM 


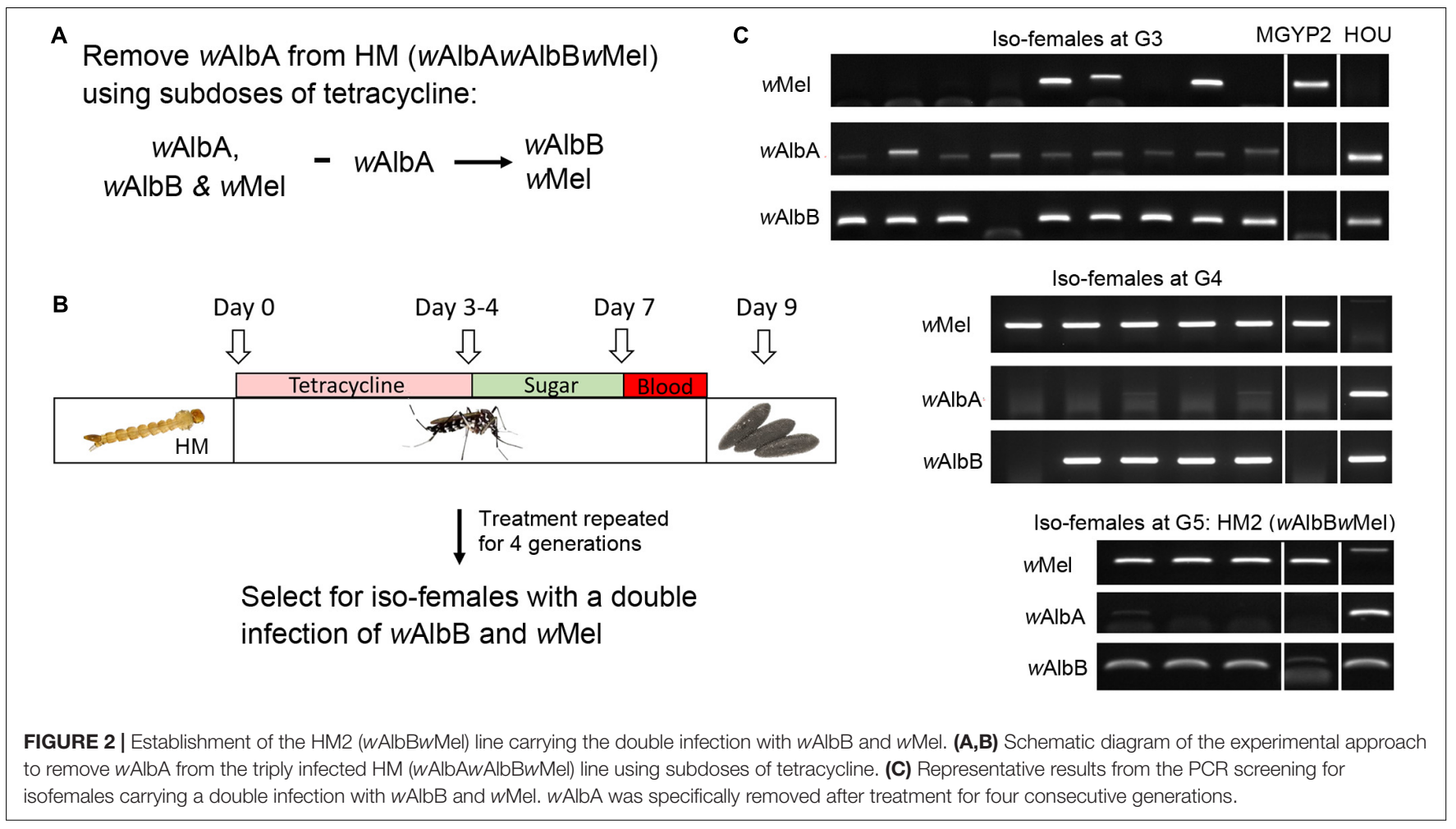

TABLE 2 | Results of $\mathrm{Cl}$ crosses among the HM2 (wAlbBwMel), GUA, HB, and HC lines.

\begin{tabular}{|c|c|c|c|c|}
\hline \multirow[t]{2}{*}{ Expected Cl type } & \multirow[t]{2}{*}{ Cross $\left(q \times 0^{2}\right)$} & \multicolumn{2}{|c|}{ Infection types } & \multirow[t]{2}{*}{ Percent egg hatch } \\
\hline & & Female & Male & \\
\hline \multirow[t]{5}{*}{ Incompatible } & $\mathrm{HM} 2 \times \mathrm{HC}$ & $w A l b B, w \mathrm{Mel}$ & wAlbA, wAlbB, wPip & $0 \pm 0 \mathrm{a}$ \\
\hline & $\mathrm{HC} \times \mathrm{HM} 2$ & wAlbA,wAlbB wPip & wAlbB, wMel & $9.0 \pm 7.8 b$ \\
\hline & $\mathrm{GUA} \times \mathrm{HM} 2$ & wAlbA, wAlbB & wAlbB, wMel & $14.6 \pm 8.3 b$ \\
\hline & $\mathrm{HM} 2 \times \mathrm{GUA}$ & $w A l b B, w \mathrm{Mel}$ & wAlbA, wAlbB & $9.3 \pm 4.2 b$ \\
\hline & $\mathrm{HB} \times \mathrm{HM} 2$ & wAlbB & wAlbB, wMel & $14.6 \pm 6.1 \mathrm{~b}$ \\
\hline \multirow[t]{5}{*}{ Compatible } & $\mathrm{HM} 2 \times \mathrm{HB}$ & wAlbB, wMel & wAlbB & $79.6 \pm 8.2 \mathrm{c}$ \\
\hline & $\mathrm{HM} 2 \times \mathrm{HM} 2$ & $w A l b B, w \mathrm{Mel}$ & $w A l b B, w \mathrm{Mel}$ & $64.8 \pm 17.7 \mathrm{~d}$ \\
\hline & $\mathrm{HB} \times \mathrm{HB}$ & $w A l b B$ & wAlbB & $83.7 \pm 6.9 c$ \\
\hline & $\mathrm{HC} \times \mathrm{HC}$ & $w A l b A, w A l b B, w P i p$ & wAlbA,wAlbB wPip & $80.8 \pm 7.8 c$ \\
\hline & $G \cup A \times G \cup A$ & wAlbA,wAlbB & wAlbA,wAlbB & $85.6 \pm 4.8 c$ \\
\hline
\end{tabular}

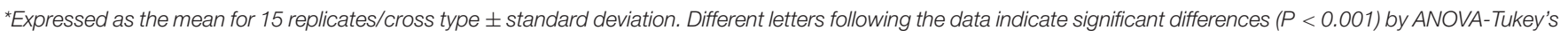
multiple comparison test.

( $w \mathrm{AlbA} w \mathrm{AlbB} w \mathrm{Mel})$ mosquitoes by qPCR. In the salivary glands at G6, the density of $w$ AlbB was significantly higher than that of $w \mathrm{AlbA}$, but there was no significant difference in density between $w \mathrm{Mel}$ and $w \mathrm{AlbA}$ or between $w \mathrm{Mel}$ and $w \mathrm{AlbB}$ (Figure 4A). In the midgut, a higher density of $w \mathrm{AlbB}$ than either $w \mathrm{Mel}$ or $w \mathrm{AlbA}$ was observed, whereas the densities of $w \mathrm{Mel}$ and $w \mathrm{AlbA}$ did not differ significantly (Figure 4B). These results indicates that $w \mathrm{AlbB}$ is dominant in the somatic tissue of $\mathrm{HM}$ ( $w \mathrm{AlbA} w \mathrm{AlbB} w \mathrm{Mel}$ ) mosquitoes. In contrast, a higher density of $w \mathrm{Mel}$ than of $w \mathrm{AlbA}$ or $w \mathrm{AlbB}$ is apparent in $\mathrm{HM}$ ( $w \mathrm{AlbA} w \mathrm{AlbB} w \mathrm{Mel}$ ) ovaries (Figure 4C). This distribution pattern was consistently maintained at G6 and G18 despite some degree of fluctuation.
To better understand the strain-specifc interactions in transinfected mosquitoes with triple infections, we also compared the densities of $w \mathrm{Pip}, w \mathrm{AlbA}$, and $w \mathrm{AlbB}$ in $\mathrm{HC}$ ovaries and observed a different order of Wolbachia density: $w$ Pip $>w$ AlbA $>w$ AlbB (Figure 4C). Consistent with previous observations (Lu et al., 2012), wAlbB was present at a higher level than was $w \mathrm{AlbA}$ in the ovaries of HOU mosquitoes, from which both the HC and HM (wAlbAwAlbBwMel) lines were originally derived (Figure 4C). We further compared the density of the same Wolbachia strain in ovaries across various mosquito lines to examine the impact of the host's genetic background on infection levels. $w$ AlbA showed its highest level of infection in $\mathrm{HM}(w \mathrm{AlbA} w \mathrm{AlbB} w \mathrm{Mel})$ ovaries at G6 but decreased by 7.8 -fold 


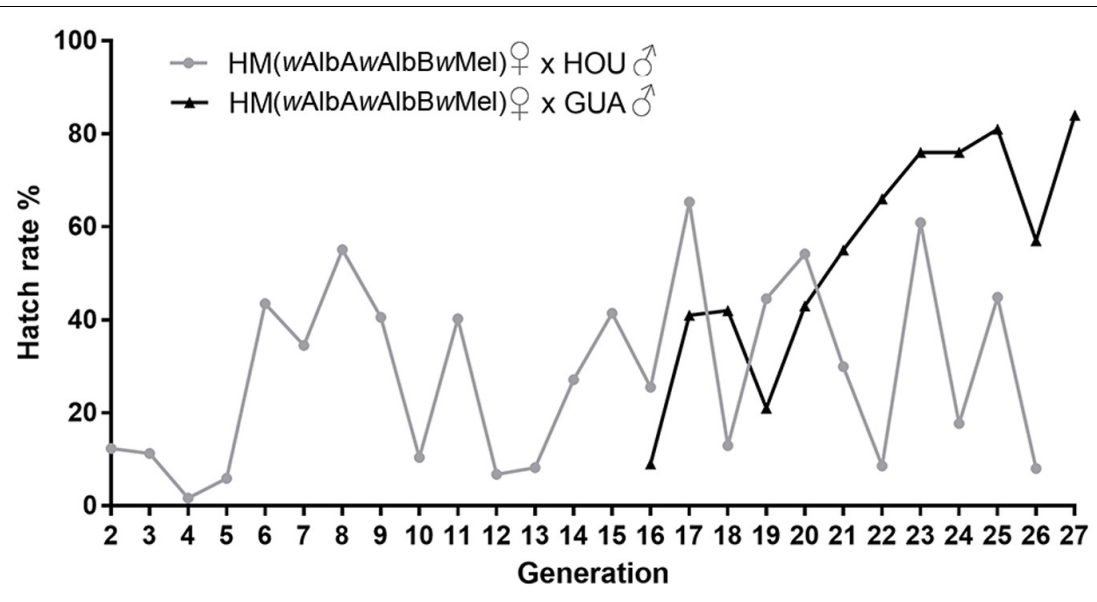

FIGURE 3 | Egg hatch rate of HM (wAlbAwAlbBwMel) females outcrossed with wild-type males from G2 to G27. Egg hatch was calculated as the percentage of eggs hatched divided by the total number of eggs (see Supplementary Table 1). Outcrosses are indicated as female $\times$ male. HM ( $w A l b A w A l b B w M e l)$, the transinfected Ae. albopictus line carrying the triple infection with wMel, wAlbA, and wAlbB. HOU and GUA, two wild-type Ae. albopictus lines carrying wAlbA and wAlbB.

at G18, when it reached a level closer to that in HOU ovaries. The density of $w$ AlbB was stable in $\mathrm{HM}$ ( $w \mathrm{AlbA} w \mathrm{AlbB} w \mathrm{Mel})$ ovaries from G6 to G18 and was consistently maintained at a level significantly higher than that in both the HOU and $\mathrm{HC}$ lines (Figure 4D). The density of $w \mathrm{Mel}$ decreased by $47 \%$ in HM ( $w$ AlbAwAlbB wMel) ovaries from G6 to G18 (Figure 4D) but was still much higher than that of the other Wolbachia strains. Interestingly, as compared to HOU ovaries, wAlbA was 1,022-fold higher in HM ( $w \mathrm{AlbA} w \mathrm{AlbB} w \mathrm{Mel}$ ) ovaries at G6, and $w$ AlbB was 1,411-fold lower in $\mathrm{HC}$ ovaries (Figure 4D). Taken together, these results indicate that Wolbachia density is regulated in triply infected Ae. albopictus in a strain-, host-, and temporally specific manner.

\section{DISCUSSION}

We have demonstrated the successful establishment of a novel triple Wolbachia infection with $w \mathrm{Mel}, w \mathrm{AlbA}$, and $w \mathrm{AlbB}$ in the Ae. albopictus HM (wAlbAwAlbBw Mel) line, with $100 \%$ maternal transmission efficiency. Experimental crosses showed that $\mathrm{CI}$ is not induced when $\mathrm{HM}$ ( $w \mathrm{AlbA} w \mathrm{AlbB} w \mathrm{Mel}$ ) males mate with either GUA or HC females, but removal of $w \mathrm{AlbA}$ from the $\mathrm{HM}$ ( $w \mathrm{AlbA} w \mathrm{AlbB} w \mathrm{Mel}$ ) mosquitoes results in $\mathrm{CI}$ when these mosquitoes are crossed with three Ae. albopictus lines carrying either a single- (HB), double- (GUA), or triple(HC) strain infection. Despite a severe reduction in the egg hatch rate associated with the triple infection, the rate was returned to normal levels by outcrossing with the wild-type GUA line, but not the HOU line. Among three different Wolbachia strains, $w \mathrm{Mel}$ and $w \mathrm{AlbB}$ were highest in density in the ovaries and midguts, respectively, of $\mathrm{HM}$ ( $w \mathrm{AlbA} w \mathrm{AlbB} w \mathrm{Mel}$ ) mosquitoes, whereas $w$ Pip and $w \mathrm{AlbB}$ were present in the highest and lowest levels, respectively, in $\mathrm{HC}$ ovaries. The densities of $w \mathrm{AlbA}$ and $w \mathrm{Mel}$, but not $w \mathrm{AlbB}$, were reduced from G6 to G18 in HM ( $w \mathrm{AlbA} w \mathrm{AlbB} w \mathrm{Mel}$ ) ovaries. These results indicate the existence of complicated interactions in term of both tissue tropism and CI expression when various Wolbachia strains co-exist in a host, providing important information to guide the design and establishment of transinfections in mosquito with optimal Wolbachia strains or their combination for disease control.

Our results indciate that competition for tissue colonization may occur between Wolbachia strains in the same supergroup. In the phylogeny of Wolbachia, both $w \mathrm{Mel}$ and $w \mathrm{AlbA}$ belong to supergroup $\mathrm{A}$, whereas $w \mathrm{AlbB}$ and $w$ Pip belong to supergroup $B$ (Werren et al., 2008). With the introduction of $w \mathrm{Mel}$ into Ae. albopictus $\mathrm{HOU}$ mosquitoes carrying $w \mathrm{AlbA}$ and $w \mathrm{AlbB}$, we observed that the density of wAlbA decreased by 7.8-fold in HM ( $w \mathrm{AlbA} w \mathrm{AlbB} w \mathrm{Mel}$ ) ovaries from $\mathrm{G} 5$ to G18, but $w \mathrm{AlbB}$ density remained stable. The level of $w \mathrm{Mel}$ infection also decreased from G5 to G18, but this decrease could have been caused by either the adaption of $w \mathrm{Mel}$ to a novel host background or competition from $w \mathrm{AlbA}$, or both. Consistent with a previous report ( $\mathrm{Lu}$ et al., 2012), the $w$ AlbB density was higher than that of $w$ AlbA in $\mathrm{HOU}$ ovaries. In the triply infected $\mathrm{HC}$ mosquitoes, generated by transfer of $w$ Pip to HOU mosquitoes (Zheng et al., 2019), wAlbB was suppressed to a minimal level in the ovaries. Specifically, the density of $w$ AlbB $\left(5.6 \times 10^{-3} w s p / r p s 6\right)$ was 7,934 - and 5,226-fold lower than that of $w \operatorname{Pip}(44.6 w s p / r p s 6)$ or $w$ AlbA (29.4 wsp/rps6), respectively. It is worth noting that this low number of $w \mathrm{AlbB}$ was still sufficient to induce $\mathrm{CI}$, given that unidirectional $\mathrm{CI}$ has been observed in crosses of HC and GUA mosquitoes (Zheng et al., 2019). Thus, when Wolbachia is being introduced into an infected host, choosing a novel strain belonging to a supergroup different from that of the orginal infection may prove useful for avoiding competition. Caution should be used if the native strain provides an essential benefit to the host, since the novel strain will likely outcompete the native strain in the transfected line, based on our observations from the $\mathrm{HM}(w \mathrm{AlbA} w \mathrm{AlbB} w \mathrm{Mel})$ and $\mathrm{HC}$ lines.

Competition for CI induction can also occur among different strains within the same supergroup. Although a single $w \mathrm{Mel}$ infection is able to induce $\mathrm{CI}$ in both Ae. aegypti and Ae. 

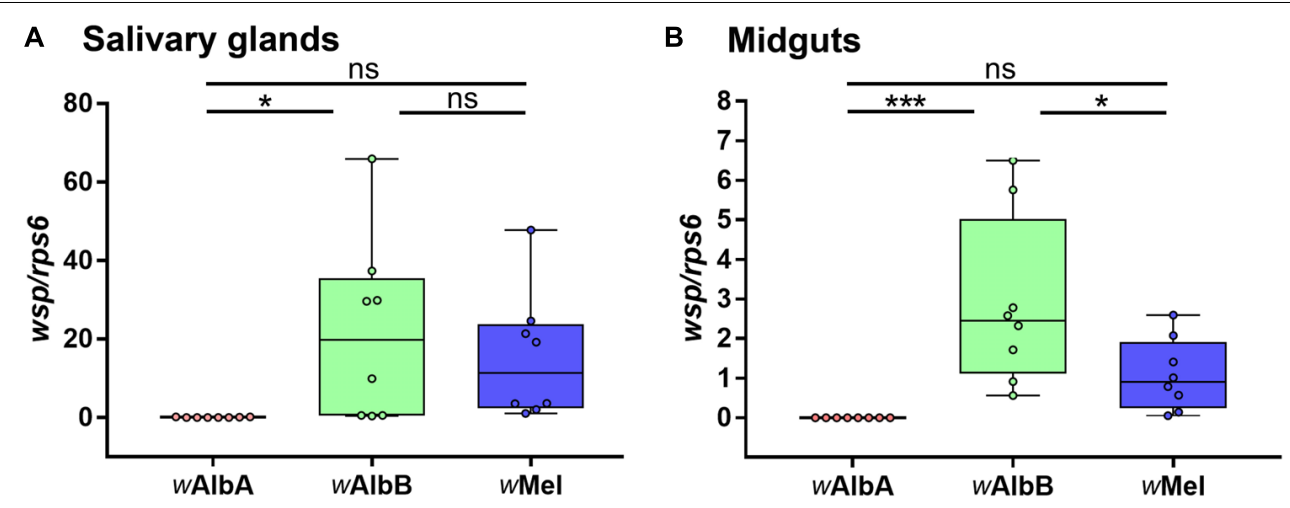

\section{Ovaries}
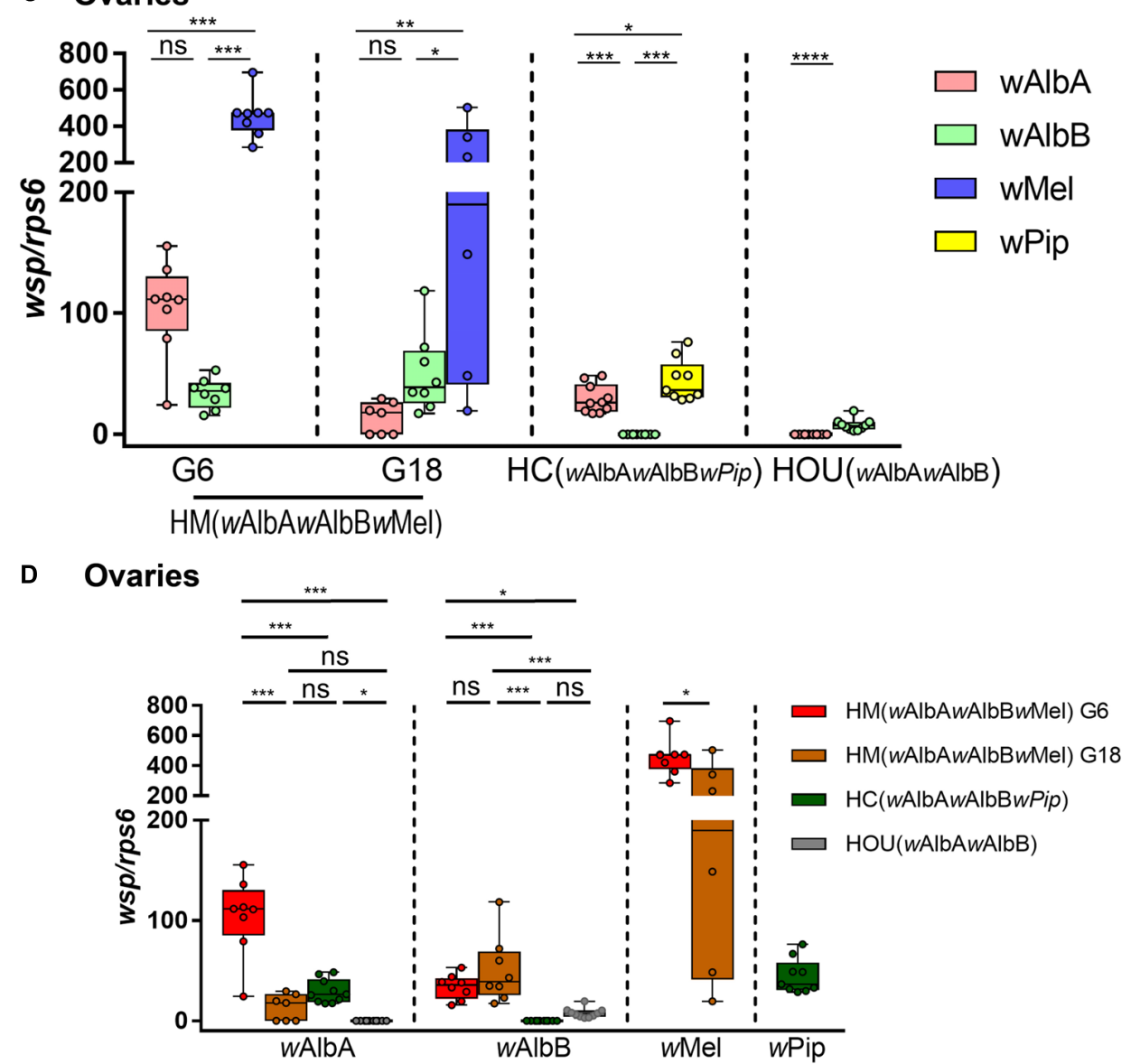

FIGURE 4 | The densities of various Wolbachia strains in the salivary glands, midguts, and ovaries of HM (wAlbAwAlbBwMel) mosquitoes. The densities of $w$ Mel, wAlbA, and wAlbB in salivary glands (A) and midguts (B) of HM (wAlbAwAlbBwMel) mosquito were measured by quantitative PCR (qPCR). The densities of the three Wolbachia strains in the ovaries of HM (wAlbAwAlbBwMel) mosquitoes from two generations, G6 and G18, were compared within (C) and across (D) mosquito lines. HC (wAlbAwAlbBwPip), the transinfected Ae. albopictus line with wAlbA, wAlbB, and wPip infections. HOU (wAlbAwAlbB), the wild-type Ae. albopictus line with wAlbA and wAlbB infections. The copy number of the Wolbachia wsp gene was normalized by the mosquito rps6 gene (see Supplementary Table 2). The center of a box plot shows the median of 6-10 replicates, edges show upper and lower quartiles, and bars indicate maximum and minimum values. Dots show values from individual biological replicates. ${ }^{\star \star \star \star} P<0.0001$; ${ }^{\star \star \star} P<0.001$; ${ }^{\star \star} P<0.01$; ${ }^{\star} P<0.05$; ns, not significant; ANOVA and Tukey's multiple comparisons test.

albopictus (Walker et al., 2011; Blagrove et al., 2012), HM ( $w \mathrm{AlbA} w \mathrm{AlbB} w \mathrm{Mel}$ ) males did not induce CI when crossed with either GUA or HC females. After removal of $w$ AlbA from the $\mathrm{HM}$ ( $w \mathrm{AlbA} w \mathrm{AlbB} w \mathrm{Mel}$ ) line, however, we observe a strong
CI expression in crosses of HM2 ( $w \mathrm{AlbB} w \mathrm{Mel})$ with either GUA, HC or HB. These results indicate that the ability of $w$ Mel to modify the HM ( $w$ AlbA $w A l b B w \mathrm{Mel}$ ) sperm may be blocked by the presence of $w$ AlbA, instead of $w$ Mel-modified 
sperm being rescued by $w$ AlbA or $w$ AlbB in $\mathrm{HC}$ or GUA mosquitoes. Consistent with our observations concerning HM2 $(w \mathrm{AlbB} w \mathrm{Mel})$ crosses, double infection of $w \mathrm{AlbB}$ and $w \mathrm{Mel}$ in transinfected Ae. aegypti was able to induce CI in the crosses with either non-infected, $w$ AlbA-, $w$ AlbB-, or $w$ Mel-infected lines (Joubert et al., 2016). A similar effort to develop a triple infection $(w \mathrm{AlbA}, w \mathrm{AlbB}$, and $w \mathrm{Mel})$ in Ae. Albopictus has been previously reported, but it resulted in very different outcomes: the triply infected line was self-incompatibility, its female was incompatible with wild-type male, and its male induced CI when crossed with wild-type females (Ant and Sinkins, 2018). It appears that the ability to rescue CI modification is compromised in their triply infected female, wherea the ability to induce CI is inhibited in our triply infected male. One possible explaination for the difference from our study is that the wAlbA density in the embryos of their triply infected line was inhibited to such an extent that it was impossible for $w$ AlbA to rescue the CI modification in the males; in contrast, in our case the infection level of $w \mathrm{AlbA}$ was not significantly reduced in the HM ( $w \mathrm{AlbA} w \mathrm{AlbB} w \mathrm{Mel}$ ) ovaries when compared to wild-type. A similar experiment with different observation indicates complicated Wolbachiahost interactions when multiple strains coexsit and stresses the importance of repeating transinfection experiments with different genetic backgrounds of both donor and recipient strains as a way to obtain a useful combination of parameters.

Blocking by $w$ AlbA of the $w$ Mel-induced modification of sperm in the $\mathrm{HM}$ ( $w \mathrm{AlbA} w \mathrm{AlbB} w \mathrm{Mel})$ line suggests a potentional competition for host targets of CI factors between $w \mathrm{Mel}$ and wAlbA. Recent studies have suggested a "two-by-one" model underlying the CI mechanism in which Wolbachia-induced sperm modification is determined by two CI factors, cif $A$ and $c$ if $B$, whereas CI rescue is determined only by cifA (LePage et al., 2017; Shropshire et al., 2018; Beckmann et al., 2019; Chen et al., 2019). Further evidence has suggested that cifB targets nuclear protein import and protamine-histone exchange and that cif $A$ rescues embryos by restricting the access of cifB to its targets (Beckmann et al., 2019). We hypothesize that the cifB genes of $w \mathrm{Mel}$ and $w$ AlbA are very similar, so that they bind to the same sites that affect the host's nuclear protein import and then are translocated to the nucleus, where their substrates for sperm modification reside. The affinity of native $w$ AlbA for host targets may be higher than that of $w \mathrm{Mel}$, thus preventing the $w \mathrm{Mel}$ from entering the nucleus to induce CI expression.

Very low rates of egg hatching were observed in the HM ( $w \mathrm{AlbA} w \mathrm{AlbB} w \mathrm{Mel}$ ) line before G6. Suprisingly, the outcross with wild-type HOU only increased egg hatch rates temporarily in some generations (e.g., G6, G17, and G23); in these cases, there was an immediate decline afterward, resulting in a fluctuation wave across 26 generations. When the HC line was initially established, low hatch rates were also observed for almost a year. The situation was different for the other transfected lines that we established, in that egg hatching quickly returned to a normal level after the outcrosses with wild-type for several consecutive generations (Xi et al., 2005a,b, 2006). It would presumably be more challenging for the host to establish a symbiotic relationship with a Wolbachia triple strain than with a single or double strain because of the overload of symbionts and the complicated interactions between various strains and the host. Interestingly, outcrosses of HM ( $w \mathrm{AlbA} w \mathrm{AlbB} w \mathrm{Mel}$ ) with another wild-type line, GUA, effectively recovered normal egg hatch rates, indicating that the GUA genetic background can facilitate the host's adaptation to the novel triple infection. Because HM ( $w$ AlbA $w$ AlbB $w \mathrm{Mel}$ ) was derived from HOU, which had been maintained for a long time in the laboratory, outcrosses with HOU may not be able to introduce as much genetic heterogeneity to foster a novel symbiosis as can outcrosses with GUA, which was recently established from field samples (Zheng et al., 2019).

Here, we have demonstrated the successful extablishment of a transinfected Ae. albopictus HM ( $w \mathrm{AlbA} w \mathrm{AlbB} w \mathrm{Mel})$ line carrying a Wolbachia triple-strain infection. Unfortunately, the newly introduced $w \mathrm{Mel}$ strain failed to induce $\mathrm{CI}$ in this triply infected line, and our experimental evidence indicates that its ability to modify the sperm was blocked by the native strain, wAlbA. Further studies are needed to compare the CI determination factors associated with $w \mathrm{Mel}$ and $w \mathrm{AlbA}$ and to understand the molecular mechanism undergirding their potential competition in utilizing host targets for CI expression. The tissue tropism of the three Wolbachia strains in the HM ( $w \mathrm{AlbA} w \mathrm{AlbB} w \mathrm{Mel})$ line indicates their complicated interactions, with competition likely to happen between Wolbachia strains in the same supergroup. The differences in both CI expression and Wolbachia tissue tropism between the two triply transinfected lines HM ( $w \mathrm{AlbA} w \mathrm{AlbB} w \mathrm{Mel})$ and $\mathrm{HC}$ also indicate that caution is necessary when predicting the outcome of transinfected lines with multiple infections. These results provide important information to guide the future selection of Wolbachia strains for the development of transinfected lines in order to obtain the maximum pathogenblocking efficiency, the lowest fitness cost, and ideal CI patterns.

\section{DATA AVAILABILITY STATEMENT}

The original contributions presented in the study are included in the article/Supplementary Material, further inquiries can be directed to the corresponding author.

\section{AUTHOR CONTRIBUTIONS}

$\mathrm{XL}$ and ZX contributed to conceptualization, methodology, validation, formal analysis, investigation, data curation, and original draft preparation. JL was responsible for CI crosses. GB performed embryo microinjection. ZX supervised the study. All authors contributed to the article and approved the submitted version.

\section{FUNDING}

This work was supported by a grant from the Foundation for the NIH through the Grand Challenges in Global Health Initiative of the Bill and Melinda Gates Foundation, Scientific and Technological Leading Talents of Guangzhou Development District (No. 2013L-P116), and a Strategic Partnership Grant from Michigan State University. 


\section{ACKNOWLEDGMENTS}

We thank S. L. O'Neill for providing MGYP2 line for this study, Xiaoling Pan and Fengrui Zhang for their technical assistance, and Dr. Deborah McClellan for editorial assistance.

\section{REFERENCES}

Achee, N. L., Gould, F., Perkins, T. A., Reiner, R. C. Jr., Morrison, A. C., et al. (2015). A critical assessment of vector control for dengue prevention. PLoS Negl. Trop. Dis. 9:e0003655. doi: 10.1371/journal.pntd.0003655

Ant, T. H., Herd, C. S., Geoghegan, V., Hoffmann, A. A., and Sinkins, S. P. (2018). The Wolbachia strain $w$ Au provides highly efficient virus transmission blocking in Aedes aegypti. PLoS Pathog. 14:e1006815. doi: 10.1371/journal.ppat.100 6815

Ant, T. H., and Sinkins, S. P. (2018). A Wolbachia triple-strain infection generates self-incompatibility in Aedes albopictus and transmission instability in Aedes aegypti. Parasit. Vect. 11, 295.

Beckmann, J. F., Sharma, G. D., Mendez, L., Chen, H., and Hochstrasser, M. (2019). The Wolbachia cytoplasmic incompatibility enzyme CidB targets nuclear import and protamine-histone exchange factors. eLife 8:e50026.

Blagrove, M. S., Arias-Goeta, C., Di Genua, C., Failloux, A. B., and Sinkins, S. P. (2013). A Wolbachia wMel transinfection in Aedes albopictus is not detrimental to host fitness and inhibits Chikungunya virus. PLoS. Negl. Trop. Dis. 7:e2152. doi: 10.1371/journal.pntd.0002152

Blagrove, M. S., Arias-Goeta, C., Failloux, A. B., and Sinkins, S. P. (2012). Wolbachia strain $w \mathrm{Mel}$ induces cytoplasmic incompatibility and blocks dengue transmission in Aedes albopictus. Proc. Natl. Acad. Sci. U.S.A. 109, 255-260. doi: $10.1073 /$ pnas. 1112021108

Chen, H., Ronau, J. A., Beckmann, J. F., and Hochstrasser, M. (2019). A Wolbachia nuclease and its binding partner provide a distinct mechanism for cytoplasmic incompatibility. Proc. Natl. Acad. Sci. U.S.A. 116, 22314-22321. doi: 10.1073/ pnas. 1914571116

Crawford, J. E., Clarke, D. W., Criswell, V., Desnoyer, M., Cornel, D., Deegan, B., et al. (2020). Efficient production of male Wolbachia-infected Aedes aegypti mosquitoes enables large-scale suppression of wild populations. Nat. Biotechnol. $38,482-492$

Fu, Y., Gavotte, L., Mercer, D. R., and Dobson, S. L. (2010). Artificial triple Wolbachia infection in Aedes albopictus yields a new pattern of unidirectional cytoplasmic incompatibility. Appl. Environ. Microbiol. 76, 5887-5891. doi: 10.1128/aem.00218-10

Hoffmann, A. A., Montgomery, B. L., Popovici, J., Iturbe-Ormaetxe, I., Johnson, P. H., Muzzi, F., et al. (2011). Successful establishment of Wolbachia in Aedes populations to suppress dengue transmission. Nature 476, 454-457. doi: 10. 1038/nature 10356

Joubert, D. A., Walker, T., Carrington, L. B., De Bruyne, J. T., Kien, D. H., Hoang Nle, T., et al. (2016). Establishment of a Wolbachia superinfection in Aedes aegypti mosquitoes as a potential approach for future resistance management. PLoS Pathog. 12:e1005434. doi: 10.1371/journal.ppat.100 5434

Katzelnick, L. C., Coloma, J., and Harris, E. (2017). Dengue: knowledge gaps, unmet needs, and research priorities. Lancet Infect. Dis. 17, e88-e100. doi: 10.1016/s1473-3099(16)30473-x

LePage, D. P., Metcalf, J. A., Bordenstein, S. R., On, J., Perlmutter, J. I., Shropshire, J. D., et al. (2017). Prophage WO genes recapitulate and enhance Wolbachiainduced cytoplasmic incompatibility. Nature 543, 243-247. doi: 10.1038/ nature21391

Lu, P., Bian, G., Pan, X., and Xi, Z. (2012). Wolbachia induces density-dependent inhibition to dengue virus in mosquito cells. PLoS Negl. Trop. Dis. 6:e1754. doi: 10.1371/journal.pntd.0001754

Mains, J. W., Brelsfoard, C. L., Rose, R. I., and Dobson, S. L. (2016). Female Adult Aedes albopictus suppression by Wolbachia-infected male mosquitoes. Sci. Rep 6:33846.

McGraw, E. A., Merritt, D. J., Droller, J. N., and O'neill, S. L. (2002). Wolbachia density and virulence attenuation after transfer into a novel host. Proc. Natl. Acad. Sci. U.S.A. 99, 2918-2923. doi: 10.1073/pnas.05246 6499

\section{SUPPLEMENTARY MATERIAL}

The Supplementary Material for this article can be found online at: https://www.frontiersin.org/articles/10.3389/fmicb. 2020.01638/full\#supplementary-material

McMeniman, C. J., Lane, R. V., Cass, B. N., Fong, A. W., Sidhu, M., Wang, Y. F., et al. (2009). Stable introduction of a life-shortening Wolbachia infection into the mosquito Aedes aegypti. Science 323, 141-144. doi: 10.1126/science.1165326

Molina-Cruz, A., Gupta, L., Richardson, J., Bennett, K., Black, W. T., and BarillasMury, C. (2005). Effect of mosquito midgut trypsin activity on dengue-2 virus infection and dissemination in Aedes aegypti. Am. J. Trop. Med. Hyg. 72, 631-637. doi: 10.4269/ajtmh.2005.72.631

Nazni, W. A., Hoffmann, A. A., Noorafizah, A., Cheong, Y. L., Mancini, M. V., Golding, N., et al. (2019). Establishment of Wolbachia strain wAlbB in malaysian populations of Aedes aegypti for dengue control. Curr. Biol. 29, 4241.e4245-4248.e4245.

Ross, P. A., Turelli, M., and Hoffmann, A. A. (2019). Evolutionary ecology of Wolbachia releases for disease control. Annu. Rev. Genet. 53, 93-116. doi: 10.1146/annurev-genet-112618-043609

Ross, P. A., Wiwatanaratanabutr, I., Axford, J. K., White, V. L., EndersbyHarshman, N. M., and Hoffmann, A. A. (2017). Wolbachia Infections in Aedes aegypti differ markedly in their response to cyclical heat stress. PLoS Pathog. 13:e1006006. doi: 10.1371/journal.ppat.1006006

Shropshire, J. D., On, J., Layton, E. M., Zhou, H., and Bordenstein, S. R. (2018). One prophage WO gene rescues cytoplasmic incompatibility in Drosophila melanogaster. Proc. Natl. Acad. Sci. U.S.A. 115, 4987-4991. doi: 10.1073/pnas. 1800650115

Suh, E., Fu, Y., Mercer, D. R., and Dobson, S. L. (2016). Interaction of Wolbachia and bloodmeal type in artificially infected Aedes albopictus (Diptera: Culicidae). J. Med. Entomol. 53, 1156-1162. doi: 10.1093/jme/tjw084

Velayudhan, R. (2012). Global Strategy for Dengue Prevention and Control, 20122020. Geneva: WHO.

Walker, T., Johnson, P. H., Moreira, L. A., Iturbe-Ormaetxe, I., Frentiu, F. D., Mcmeniman, C. J., et al. (2011). The wMel Wolbachia strain blocks dengue and invades caged Aedes aegypti populations. Nature 476, 450-453. doi: 10.1038/ nature 10355

Werren, J. H., Baldo, L., and Clark, M. E. (2008). Wolbachia: master manipulators of invertebrate biology. Nat. Rev. Microbiol. 6, 741-751. doi: 10.1038/ nrmicro1969

Xi, Z., Dean, J. L., Khoo, C., and Dobson, S. L. (2005a). Generation of a novel Wolbachia infection in Aedes albopictus (Asian tiger mosquito) via embryonic microinjection. Insect. Biochem. Mol. Biol. 35, 903-910. doi: 10.1016/j.ibmb. 2005.03.015

Xi, Z., Khoo, C. C., and Dobson, S. L. (2005b). Wolbachia establishment and invasion in an Aedes aegypti laboratory population. Science 310, 326-328. doi: $10.1126 /$ science. 1117607

Xi, Z., Khoo, C. C., and Dobson, S. L. (2006). Interspecific transfer of Wolbachia into the mosquito disease vector Aedes albopictus. Proc. Biol. Sci. 273, $1317-$ 1322. doi: $10.1098 / \mathrm{rspb} .2005 .3405$

Zheng, X., Zhang, D., Li, Y., Yang, C., Wu, Y., Liang, X., et al. (2019). Incompatible and sterile insect techniques combined eliminate mosquitoes. Nature 572, 56-61. doi: 10.1038/s41586-019-1407-9

Conflict of Interest: JL and ZX were employed by Guangzhou Wolbaki Biotech Co., Ltd.

The remaining authors declare that the research was conducted in the absence of any commercial or financial relationships that could be construed as a potential conflict of interest.

Copyright (c) 2020 Liang, Liu, Bian and Xi. This is an open-access article distributed under the terms of the Creative Commons Attribution License (CC BY). The use, distribution or reproduction in other forums is permitted, provided the original author(s) and the copyright owner(s) are credited and that the original publication in this journal is cited, in accordance with accepted academic practice. No use, distribution or reproduction is permitted which does not comply with these terms. 